\title{
Mental health of medical workers in Japan during COVID-19: Relationships with loneliness, hope and self-compassion
}

\author{
Yasuhiro Kotera $^{1}$ (D) Akihiko Ozaki ${ }^{2,3} \cdot$ Hirotomo Miyatake $^{4} \cdot$ Chie Tsunetoshi $^{4} \cdot$ Yoshitaka Nishikawa $^{5} \cdot$ \\ Tetsuya Tanimoto ${ }^{3}$
}

Accepted: 16 February 2021 / Published online: 20 February 2021

(C) The Author(s) 2021

\begin{abstract}
The current pandemic of the coronavirus disease 2019 (COVID-19) has negatively impacted medical workers' mental health in many countries including Japan. Although research identified poor mental health of medical workers in COVID-19, protective factors for their mental health remain to be appraised. Accordingly, this study aimed to investigate relationships between mental health problems, loneliness, hope and self-compassion among Japanese medical workers, and compare with the general population. Online self-report measures regarding those four constructs were completed by 142 medical workers and 138 individuals in the general population. T-tests and multiple regression analysis were performed. Medical workers had higher levels of mental health problems and loneliness, and lower levels of hope and self-compassion than the general population. Loneliness was the strongest predictor of mental health problems in the medical workers. Findings suggest that Japanese medical workplaces may benefit from targeting workplace loneliness to prevent mental health problems among the medical staff.
\end{abstract}

Keywords Japan $\cdot$ Medical workers $\cdot$ Mental health $\cdot$ Loneliness $\cdot$ COVID-19

\section{Introduction}

Poor psychological status among medical workers can limit the quality and quantity of the medical workforce, leading to poor clinical outcomes of patients. This is particularly relevant to the current pandemic of the novel coronavirus disease 2019 (COVID-19), as it can cause negative impacts on mental health of medical workers (Matsuo et al., 2020; Moreno et al., 2020; Spoorthy et al., 2020). Considering strong stigma attached to mental illnesses among Japanese medical professionals (Ando, Yamaguchi, Aoki, \& Thornicroft, 2013), directly engaging with mental health problems may not be effective as it can stimulate their stigma (Kotera et al., 2021). Previous studies elucidated the prevalence and levels of mental distress in medical workers during COVID-19 (Matsuo

Yasuhiro Kotera

Y.Kotera@derby.ac.uk

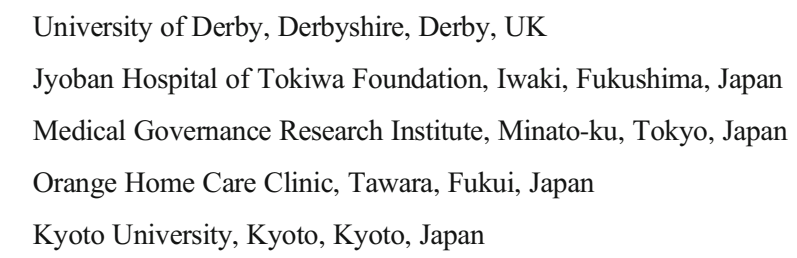

et al., 2020; Moreno et al., 2020; Spoorthy et al., 2020), however protective factors for their mental health were not evaluated. Healthful factors of mental health need to be appraised to identify effective interventions (Choi et al., 2020). Accordingly, we examined the relationships between mental health problems (depression and anxiety), loneliness (feeling alienated from others; Sekhon \& Srivastava, 2019), hope (positive motivational construct, helpful during a crisis; Bernardo \& Mendoza, 2020), and self-compassion (kindness towards oneself, associated with wellbeing; Sinclair, Kondejewski, Raffin-Bouchal, King-Shier, \& Singh, 2017) during the COVID-19 pandemic in medical workers in Japan to appraise protective factors of mental health that are unique to this population, and suggest effective approaches.

\section{Methods}

We aimed to contextualise the psychological status of medical workers by comparing with that of the general population in Japan. Online survey was distributed to Facebook groups formed by medical workers and the general population in June 2020, to which opportunity samples of 160 medical workers and 164 individuals in the general population agreed to participate. Participants had to (i) be 18 years old or older, 
(ii) be living in Japan at the time of the study, and (iii) have at least three years of experience living in Japan. To reduce the workload of participants, following four short scales were included in the survey: The Patient Health Questionnaire-4 (Löwe et al., 2010), Three-Item Loneliness Scale (Hughes, Waite, Hawkley, \& Cacioppo, 2004), Adult State Hope Scale (Snyder et al., 1996), and Self-Compassion ScaleShort Form (Raes, Pommier, Neff, \& Van Gucht, 2011). Of the agreed participants, 142 medical workers (28 doctors, 34 nurses, 29 pharmacists, 27 rehabilitation workers, and the remaining 24 included social workers and radiographers) and 138 individuals from the general population (85 full-time employees, 29 self-employed workers, 11 part-timers, and the remaining 13 included unemployed and homemakers) completed the survey (Table 1). Both groups satisfied the required sample size per as power calculation (119: Effect size $\mathrm{f}^{2}=$ 0.15, $\alpha=0.05$, Power =0.95; (Faul, Erdfelder, Buchner, \& Lang, 2009). Once data was screened for outliers and normalities, t-tests and multiple regression analysis were conducted. Ethical approval was obtained from the university research ethics committee. This study followed the Strengthening the Reporting of Observational Studies in Epidemiology (STROBE) reporting guideline.

\section{Results}

Our t-tests revealed that medical workers had high levels of mental health problems $(p=0.004, t=2.88)$ and loneliness $(p=0.043, t=2.04)$, and low levels of hope $(p=0.010, t=$
$-2.62)$ and self-compassion $(p=0.004, t=-2.89)$, relative to the general population.

In multiple regression, first, gender and age were entered to adjust for their effects (Step 1), then, loneliness, hope and selfcompassion scores were entered (Step 2). Multicollinearity was of no concern (Variance Inflation Factors $<10$ ). Loneliness $(p<0.001, \beta=0.39)$, hope $(p<0.001, \beta=$ $-0.28)$ and self-compassion $(p=0.007, \beta=-0.22)$ were significant predictors of mental health problems in medical workers, where loneliness was the strongest, and selfcompassion was the weakest predictor; whereas hope was the strongest in the general population $(p=0.003, \beta=-0.27)$ (Table 2). The three predictors predicted a greater variance of mental health problems in medical workers than in the general population $(44 \%>30 \%)$.

\section{Discussion}

During the COVID-19 pandemic in Japan, our analyses identified (i) poor mental health and weakened psychological resources of medical staff, and (ii) the importance of loneliness, hope and self-compassion to their mental health, particularly the strongest impact of loneliness. These findings suggest that the medical workforce in Japan can benefit from targeting loneliness in the workplace. As increasingly uncovered in occupational psychology, workplace loneliness is associated with limited job performance (Ozcelik \& Barsade, 2018). Among the loneliness interventions, re-appraising maladaptive social cognitions of lonelier workers, conducted regularly, was found

Table 1 Participants characteristics and psychological variables

\begin{tabular}{|c|c|c|c|c|}
\hline & \multicolumn{2}{|l|}{ Medical Workers $(n=142)$} & \multicolumn{2}{|l|}{ General Population $(n=138)$} \\
\hline & M & SD & M & SD \\
\hline \multicolumn{5}{|l|}{ Characteristics } \\
\hline Gender & F: $73 \%(n=104), \mathrm{M}=27 \%(n=38)$ & & $\mathrm{F}: 61 \%(n=84), \mathrm{M}: 39 \%(n=54)$ & \\
\hline Age & 39.90 & 12.10 & $46.39 * *$ & 10.35 \\
\hline \multirow[t]{6}{*}{ Job Role / Employment Status } & Doctors & 28 & Full-Time Employees & 85 \\
\hline & Nurses & 34 & Part-Timers & 11 \\
\hline & Pharmacists & 29 & Self-Employed incl. Employers & 29 \\
\hline & Rehabilitation Workers & 27 & Homemakers & 5 \\
\hline & Social Workers & 5 & Unemployed & 2 \\
\hline & Others incl. Nutritionists, Radiographers & 19 & Others incl. Students & 6 \\
\hline \multicolumn{5}{|l|}{ Psychological Variables } \\
\hline Mental Health Problems (measured by PHQ-4) & $3.27 * *$ & 2.78 & 2.37 & 2.31 \\
\hline Loneliness & $4.73 *$ & 1.7 & 4.33 & 1.56 \\
\hline Hope & 29.85 & 8.46 & $32.35 * *$ & 7.82 \\
\hline Self-Compassion & 3.13 & 0.58 & $3.34 * *$ & 0.61 \\
\hline
\end{tabular}

${ }^{*} p<.05, * * p<.01$ Significant difference between the two groups (noted on the higher value) 
Table 2 Multiple regression analysis for mental health problems

\begin{tabular}{|c|c|c|c|c|c|c|c|c|c|c|}
\hline \multirow[b]{3}{*}{ Step 1} & \multicolumn{5}{|c|}{ Medical Workers } & \multicolumn{5}{|c|}{ General Population } \\
\hline & \multirow[t]{2}{*}{ B } & \multirow[t]{2}{*}{$\mathrm{SE}_{\mathrm{B}}$} & \multirow[t]{2}{*}{$\beta$} & \multicolumn{2}{|c|}{$95.0 \%$ CI for B } & \multirow[t]{2}{*}{ B } & \multirow[t]{2}{*}{$\mathrm{SE}_{\mathrm{B}}$} & \multirow[t]{2}{*}{$\beta$} & \multicolumn{2}{|c|}{$95.0 \% \mathrm{CI}$ for B } \\
\hline & & & & Lower & Upper & & & & Lower & Upper \\
\hline Gender & 0.03 & 0.17 & 0.02 & -0.31 & 0.37 & -0.18 & 0.14 & -0.11 & -0.45 & 0.09 \\
\hline Age & -0.01 & 0.01 & -0.13 & -0.02 & 0.00 & -0.01 & 0.01 & -0.12 & -0.02 & 0.00 \\
\hline Adj. $\mathrm{R}^{2}$ & $0.30 \%$ & & & & & $1 \%$ & & & & \\
\hline \multicolumn{11}{|l|}{ Step 2} \\
\hline Gender & 0.09 & 0.13 & 0.05 & -0.17 & 0.35 & -0.11 & 0.12 & -0.07 & -0.34 & 0.12 \\
\hline Age & 0.01 & 0.01 & 0.09 & 0.00 & 0.02 & 0.00 & 0.01 & -0.03 & -0.02 & 0.01 \\
\hline Loneliness & 0.92 & 0.17 & $0.39 * * *$ & 0.58 & 1.25 & 0.50 & 0.25 & $0.20 *$ & 0.01 & 0.99 \\
\hline Hope & -0.31 & 0.08 & $-0.28 * * *$ & -0.47 & -0.14 & -0.33 & 0.11 & $-0.27 * *$ & -0.54 & -0.11 \\
\hline Self-Compassion & -1.17 & 0.42 & $-0.22 * *$ & -2.01 & -0.33 & -1.06 & 0.49 & $-0.21^{*}$ & -2.04 & -0.09 \\
\hline$\Delta$ Adj. $\mathrm{R}^{2}$ & $44 \%$ & & & & & $30 \%$ & & & & \\
\hline
\end{tabular}

Outcome variable Mental Health Problems. $B$ Unstandardized Coefficients, $S E_{B}$ Standard Error of the Coefficient, $\beta$ Standardized Coefficients. $* p<0.05 ; * * p<0.01 ; * * * p<0.001$

most effective (Masi, Chen, Hawkley, \& Cacioppo, 2011), translating self-criticism into self-compassion. A caution is required as a concept of loneliness differs between individualistic and collective cultures, and these studies were primarily conducted in the Western individualistic contexts, different from the Japanese collectivism (Heu, van Zomeren, \& Hansen, 2019). Moreover, our study used (a) self-report measures, susceptible to response biases (Kotera et al., 2020) and (b) a crosssectional design, unable to elucidate causality of the variableslongitudinal studies should be conducted. Still, our findings would help identify practical interventions to be employed by Japanese medical teams during COVID-19. As the third wave has arrived in Japan in November 2020 (Kami, 2020), Japanese medical workers need to protect their mental health to continue to offer quality care for ever-increasing patients suffering from this fatal disease.

Author Contributions Study concept and design: 1st Author; Acquisition and preparation of the dataset: 1st to 5th Authors; Statistical analysis: $1 \mathrm{st}$ and 2nd Authors; Interpretation of the data: All authors; Drafting of the manuscript: 1st, 2nd and 6th Authors; Critical revision of the manuscript for important intellectual content: All authors. 1st Author had full access to all of the data in the study and takes responsibility for the integrity of the data and the accuracy of the data analysis. All authors saw and agreed on the final manuscript as well as the decision to submit for publication.

Availability of Data and Material The data that support the findings of this study are available from the corresponding author upon reasonable request.

Code Availability N/A

\section{Declarations}

Ethics Approval The 1st author's university research ethics committee has approved this study.
Consent to Participate All study participants consented to participate to the study.

Consent for Publication All authors consented to the publication of this article.

Conflict of Interest No conflicts of interested to be noted.

Open Access This article is licensed under a Creative Commons Attribution 4.0 International License, which permits use, sharing, adaptation, distribution and reproduction in any medium or format, as long as you give appropriate credit to the original author(s) and the source, provide a link to the Creative Commons licence, and indicate if changes were made. The images or other third party material in this article are included in the article's Creative Commons licence, unless indicated otherwise in a credit line to the material. If material is not included in the article's Creative Commons licence and your intended use is not permitted by statutory regulation or exceeds the permitted use, you will need to obtain permission directly from the copyright holder. To view a copy of this licence, visit http://creativecommons.org/licenses/by/4.0/.

\section{References}

Ando, S., Yamaguchi, S., Aoki, Y., \& Thornicroft, G. (2013). Review of mental-health-related stigma in Japan. Psychiatry and Clinical Neurosciences, 67(7), 471-482. https://doi.org/10.1111/pcn.12086.

Bernardo, A. B. I., \& Mendoza, N. B. (2020). Measuring hope during the COVID-19 outbreak in the Philippines: Development and validation of the state locus-of-Hope scale short form in Filipino. Current Psychology, 1-10. https://doi.org/10.1007/s12144-020-00887-x.

Choi, K. W., Ph, D., Stein, M. B., Nishimi, K. M., Ph, D., Ge, T., Ph, D., Coleman, J. R. I., \& Ph, D. (2020). An exposure-wide and mendelian randomization approach to identifying modifiable factors for the prevention of depression. 8, 1-11. https://doi.org/10.1176/appi. ajp.2020.19111158.

Faul, F., Erdfelder, E., Buchner, A., \& Lang, A.-G. (2009). Statistical power analyses using $G^{*}$ power 3.1: Tests for correlation and 
regression analyses. Behavior Research Methods, 41(4), 11491160. https://doi.org/10.3758/BRM.41.4.1149.

Heu, L. C., van Zomeren, M., \& Hansen, N. (2019). Lonely alone or lonely together? A cultural-psychological examination of individualism-collectivism and loneliness in five European countries. Personality and Social Psychology Bulletin, 45(5), 780-793. https://doi.org/10.1177/0146167218796793.

Hughes, M. E., Waite, L. J., Hawkley, L. C., \& Cacioppo, J. T. (2004). A short scale for measuring loneliness in large surveys: Results from two population-based studies. Research on Aging, 26(6), 655-672. https://doi.org/10.1177/0164027504268574.

Kami, M. (2020, November 25). Third wave of COVID-19 in Japan: What's missing in our policies? Toyokeizai. https://toyokeizai.net/ articles/-/390991. Accessed 15 Feb 2021.

Kotera, Y., Van Laethem, M., Ohshima, R. (2020). Cross-cultural comparison of mental health between Japanese and Dutch workers: relationships with mental health shame, self-compassion, work engagement and motivation. Cross Cultural \& Strategic Management, 27, (3): 511-530.

Kotera, Y., Green, P. \& Sheffield, D. (2021). Positive psychology for mental wellbeing of UK therapeutic students: Relationships with engagement, motivation, resilience, and self-compassion. International Journal of Mental Health and Addiction. https://doi.org/10.1007/ s11469-020-00466-y.

Löwe, B., Wahl, I., Rose, M., Spitzer, C., Glaesmer, H., Wingenfeld, K., Schneider, A., \& Brähler, E. (2010). A 4-item measure of depression and anxiety: Validation and standardization of the patient health Questionnaire-4 (PHQ-4) in the general population. Journal of Affective Disorders, 122(1-2), 86-95. https://doi.org/10.1016/j.jad. 2009.06.019.

Masi, C. M., Chen, H. Y., Hawkley, L. C., \& Cacioppo, J. T. (2011). A meta-analysis of interventions to reduce loneliness. Personality and Social Psychology Review, 15(3), 219-266. https://doi.org/10.1177/ 1088868310377394.

Matsuo, T., Kobayashi, D., Taki, F., Sakamoto, F., Uehara, Y., Mori, N., \& Fukui, T. (2020). Prevalence of health care worker nurnout during the coronavirus disease 2019 (COVID-19) pandemic in Japan.
JAMA Network Open, 3(8), e2017271. https://doi.org/10.1001/ jamanetworkopen.2020.17271.

Moreno, C., Wykes, T., Galderisi, S., Nordentoft, M., Crossley, N., Jones, N., Cannon, M., Correll, C. U., Byrne, L., Carr, S., Chen, E. Y. H., Gorwood, P., Johnson, S., Kärkkäinen, H., Krystal, J. H., Lee, J., Lieberman, J., López-Jaramillo, C., Männikkö, M., Phillips, M. R., Uchida, H., Vieta, E., Vita, A., \& Arango, C. (2020). How mental health care should change as a consequence of the COVID19 pandemic. The Lancet Psychiatry., 7, 813-824. https://doi.org/ 10.1016/S2215-0366(20)30307-2.

Ozcelik, H., \& Barsade, S. G. (2018). No employee an island: Workplace loneliness and job performance. Academy of Management Journal, 61(6), 2343-2366. https://doi.org/10.5465/amj.2015.1066.

Raes, F., Pommier, E., Neff, K. D., \& Van Gucht, D. (2011). Construction and factorial validation of a short form of the selfcompassion scale. Clinical Psychology \& Psychotherapy, 18(3), 250-255. https://doi.org/10.1002/cpp.702.

Sekhon, S. K., \& Srivastava, M. (2019). Conquering workplace loneliness individual or organization accountability. Human Resource Management International Digest, 27(1), 1-3. https://doi.org/10. 1108/HRMID-09-2018-0182.

Sinclair, S., Kondejewski, J., Raffin-Bouchal, S., King-Shier, K. M., \& Singh, P. (2017). Can self-compassion promote healthcare provider well-being and compassionate care to others? Results of a systematic review. Applied Psychology. Health and Well-Being, 9(2), 168206. https://doi.org/10.1111/aphw.12086.

Snyder, C. R., Sympson, S. C., Ybasco, F. C., Borders, T. F., Babyak, M. A., \& Higgins, R. L. (1996). Development and validation of the state Hope scale. Journal of Personality and Social Psychology, 70(2), 321-335. https://doi.org/10.1037/0022-3514.70.2.321.

Spoorthy, M. S., Pratapa, S. K., Mahant, Supriya. (2020). Mental health problems faced by healthcare workers due to the COVID-19 pandemic-A review. Asian Journal of Psychiatry, 51, 102119

Publisher's Note Springer Nature remains neutral with regard to jurisdictional claims in published maps and institutional affiliations. 